\title{
A fully biocatalytic approach to angiopterlactone B based on a chemoinspired artificial in vitro metabolism
}

\author{
Alexander F. Kiefer, a,b Yu-Chang Liu, ${ }^{a}$ Rebecca Gummerer, ${ }^{a}$ Christina Jäger, a and Jan Deska a* \\ (a) Department of Chemistry, Aalto University, Espoo, Finland \\ (b) Helmholtz Institute for Pharmaceutical Research Saarland, Saarbrücken, Germany \\ jan.deska@aalto.fi
}

Nature's way to construct highly complex molecular
entities with virtue as part of biosynthetic pathways is
unmatched by any chemical synthesis. Yet, relying on a
cascade of native enzymatic transformations to achieve
a certain target structure, biosynthesis is also
significantly limited in its scope. In this work, non-
natural biocatalytic modules are successfully
implemented into an artificial metabolism, combining
the benefits of traditional retrosynthesis with the
elegance and efficacy of biosynthetic networks. In a
highly streamlined process, a fully enzymatic total
synthesis of the tricyclic angiopterlactone B is achieved.

Enzymatic catalysis is nowadays often considered as an optimal template for the development of chemical processes, and imitations of the natural activation modes have led to numerous successful and broadly applied methodologies in the fields of organic and pharmaceutical chemistry.[1-5] Likewise, biocatalysis itself has been established as a powerful tool for the synthesis of organic compounds and today we can rely on an ever growing toolbox that assists synthetic chemists to develop routes based on a biocatalytic retrosynthesis.[6] Nevertheless, two centuries of chemical research have not only led to biomimetic approaches to solve synthetic challenges but also created a parallel universe of powerful molecular transformations. More recently, various research groups worldwide have started to investigate a portfolio of nonnatural enzymatic reactions such as the insertions of carbenes into $\mathrm{C}-\mathrm{H}$ bonds, [7] metathesis[8] and cyclopropanations [9] in an attempt to reverse the biomimetics approach by imitating chemistry with biological tools. Particularly by using these abiotic enzymes for the implementation in enzyme cascades and thus the design of artificial pathways based on non-natural reactions would be a bright opportunity to bring the biochemical machinery closer to the actual demands in academia and chemical industry. By arranging several enzymes in series, the overall efficiency of a multistep synthesis sequence can thus be significantly improved, since intermediate purification steps can be avoided especially when dealing with unstable derivatives, while saving resources and time.[10,11] The specific aim of this study is showcase for the enormous synthetic potential of implementing the extended reaction portfolio provided by abiotic biocatalysis modules, by presenting a streamlined multi-enzyme cascade for the total synthesis of the structurally unique angiopterlactone $\mathrm{B}$.

The angiopterlactones were isolated during a screening campaign of bioactive plant compounds from the rhizome of Angiopteris caudatiformis, a fern species that grows primarily in Asia.[12] The tricyclic core structure of angiopterlactone B comprises seven stereogenic centers and may potentially be derived from the co-isolated minor compound angiopterlactone A through an intramolecular
Michael addition. ${ }^{[13]}$ Furthermore, their bioactivity profile has not yet been fully elucidated, thus a flexible synthetic route to the natural product and its analogues would be highly desirable regarding structure-activity relationship studies to identify potential targets of this unexplored compound class.

Recently, the group of Lawrence published the first total synthesis of one of the lactones utilizing traditional synthetic transformations (Figure 1a).[13] Taking the Lawrence route as an effective and highly elegant chemical blueprint, we present herein a streamlined, chemo-inspired artificial metabolism to convert simple biogenic furans to the highly complex target lactone 5 (Figure 1b). In addition to native enzymatic conversions, a series of artificial modules for completely abiotic transformations, that is, an Achmatowicz ring expansion and a stereoselective borrowing hydrogen redox isomerization, are combined in an integrated process. As our investigation also underlines that the intermediate osmundalactone can be directly converted to the tricyclic angiopterlacone B in the aqueous

a Total synthesis applying traditional chemistry tools (Lawrence, 2017)

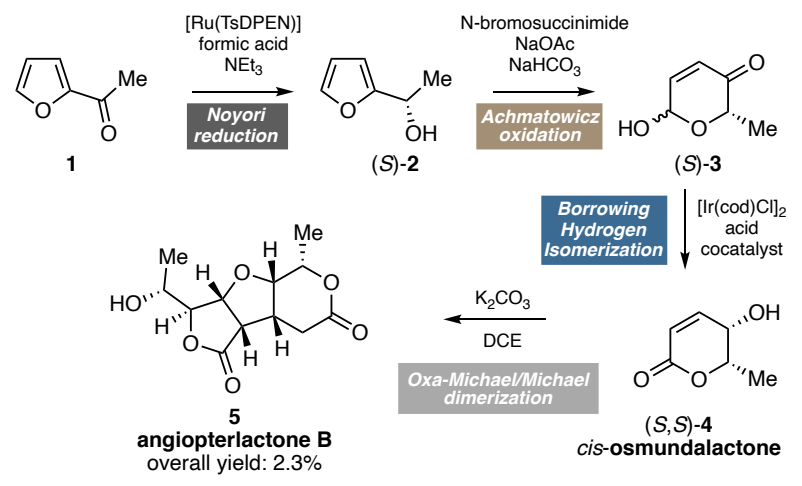

b Chemoinspired design of a fully integrated artificial metabolism (this work)

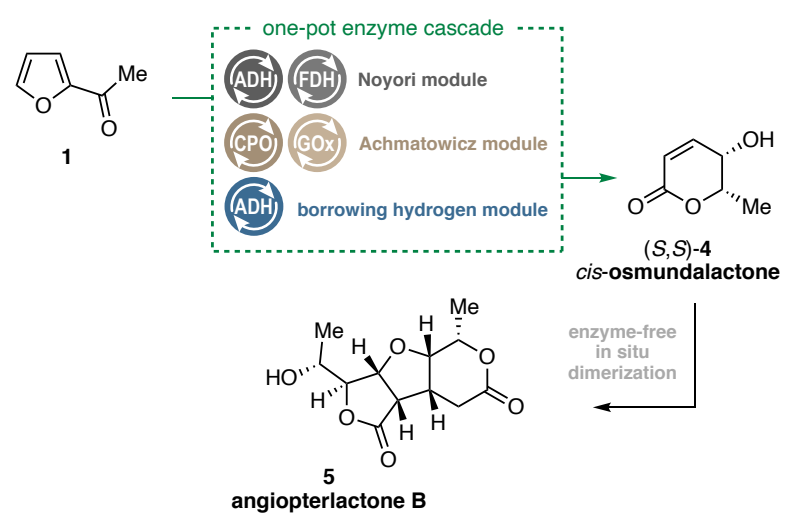

Figure 1 | Total synthesis of angiopterlactone B: traditional synthetic chemistry (a) vs integrated biocascades (b). 
reaction medium in a stereoselective manner, implications on the biosynthetic origin (genetically encoded vs spontaneous dimerization) can be derived. Overall, this case study introduces an unprecedented concept where traditional chemical retrosynthesis is translated in its entirety into an enzyme-based cascade design. In addition to the intrinsic benefits of biocatalytic cascades such as improved step economy and benign reaction conditions, this integrated artificial metabolism exceeds the efficiency of its chemical blueprint spectacularly, with a more than 10fold overall yield improvement.

For the effective translation of Lawrence's elegant and short synthesis route of angiopterlactone B into a fully enzyme-based scenario, generally all individual modules had already been developed over the past years.[14-17] Nevertheless, aiming for a true one-pot biocatalytic cascade, a number of restrictions and potential pitfalls have been identified (Figure 2). Therefore, the successful implementation of the artificial metabolism requires a very thorough understanding of catalytic parameters, interferences and dependencies which will be discussed indepth in the following paragraphs.

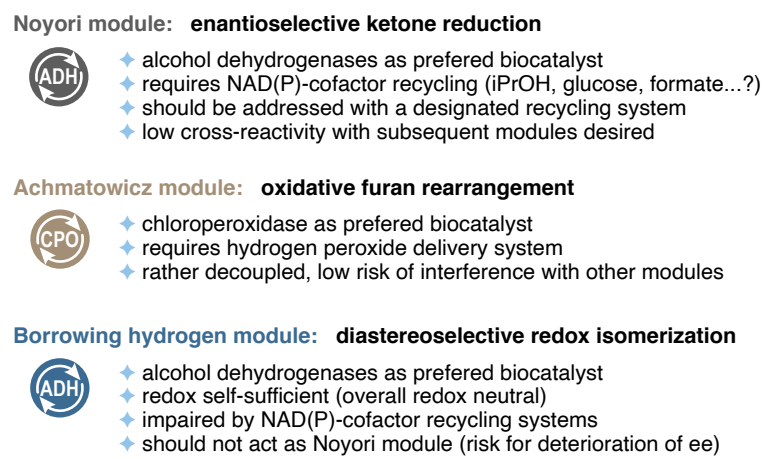

Borrowing hydrogen module: diastereoselective redox isomerization

$\checkmark$ alcohol dehydrogenases as prefered biocatalyst

$\checkmark$ redox self-sufficient (overall redox neutral)

$\rightarrow$ impaired by $N A D(P)$-cofactor recycling system

s should not act as Noyori module (risk for deterioration of ee)

Figure 2 | Key characteristics \& challenges of individual modules

With regard to scalability and optimization towards a one-pot protocol, we first required sufficient amounts of the enantiomerically enriched first intermediate 2, which should be used to obtain the complex tricyclic natural products. Thus, firstly acetylfuran $\mathbf{1}$ had to be converted to the corresponding chiral furylethanol 2. Traditionally, chiral metal catalysts are used, but they are often expensive and suffer from low turnovers and enantioselectivities. To overcome these drawbacks, two biocatalytic alternatives with mild environmentally friendly reaction conditions can be envisaged for the asymmetric reduction of ketones. This includes the kinetic resolution of racemic alcohols, restricted to a theoretical yield of $50 \%$ for desired enantiomer, while the asymmetrical reduction via alcohol dehydrogenases (ADHs) enables a theoretical substrate conversion of $100 \%$. In the latter the limiting cofactor recycling of $\mathrm{NAD}(\mathrm{P}) \mathrm{H}$, can easily be remedied by the use of multi-enzyme systems, either substrate-coupled or enzyme-coupled, to improve an in situ cofactor regeneration. To our delight, the asymmetric reduction protocol using ADHs and an NAD(P)+-dependent glucose 1dehydrogenase proved to be very stable and reliable (Figure 3a). From a set of 26 commercial biocatalysts taken from the Codexis Inc. and evoCatal GmbH ketoreductase screening kits, a total of 13 enzymes showed significant activity and led to the formation of the desired chiral secondary alcohol $\mathbf{2}$ in acceptable to excellent conversions. Closer inspection showed that seven of the tested biocatalysts provided synthetically useful stereoinduction upon ketone reduction of $\mathbf{1}$, resulting in the optically highly enriched alcohol 2 with an enantiomeric excess $>97 \%$. Alongside with five $R$-selective ADHs (P1-A04: 98\% ee, P1H10: 98\% ee, P2-H07: 98\% ee, P2-G03: 97\% ee, NADH110: $97 \% e e$ ), two enzymes with decent enantiocomplementary $S$-selectivity (NADH101: 98\% ee, evo030: 99\% ee) could be identified. The subsequent combination with the previously established Achmatowicz protocol [14] based on glucose oxidase and chloroperoxidase led to two highly effective four-enzyme one-pot systems giving rise pyranone 3 . Employing either NADH110 or evo030 as reductive module, a smooth quantitative conversion of acetylfuran 1 to either of the enantiomers of the corresponding six-membered heterocycles $(R)-\mathbf{3}$ and $(S)-3$ was achieved without any pronounced mismatched effects.

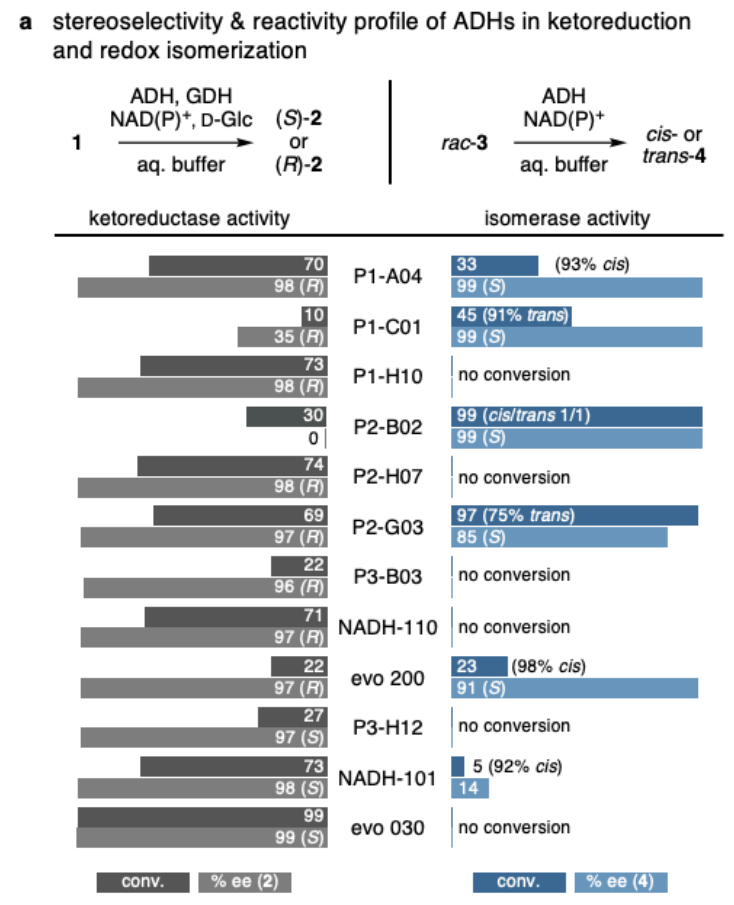

b Cofactor specificity: NADH vs NADPH

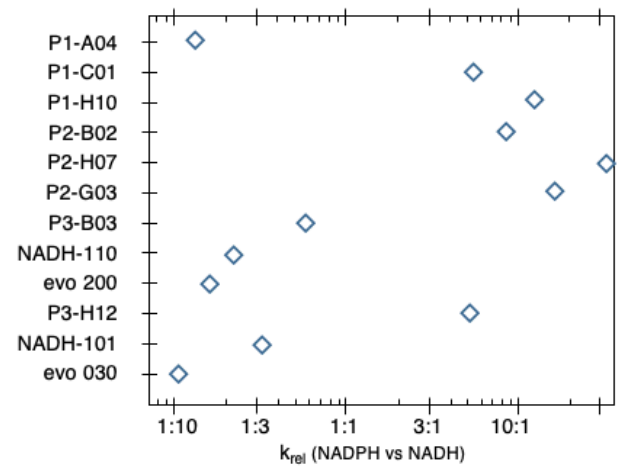

Figure 3 | Characterization of alcohol dehydrogenases to identify potentially productive binary combinations for the multi-enzyme cascades.

To evaluate the second artificial step, all seven isomerization-active $\mathrm{ADH}$ had to be more closely investigated prior to the implementation into a cascade system. On the one hand, it was necessary to assess the influence of the preferred ADHs on the redox behavior with respect to the Achmatowicz pyranones and, on the other hand, to ensure that the isomerization-active ADHs P1-C01 and P2-B02 did not exhibit a negative influence during the first reduction step. To investigate the borrowing hydrogen 
catalysis of Achmatowicz pyranones to hydroxylated $\delta$ lactones (4), the racemic hydroxy-methylpyranone rac-3 was reacted with the corresponding ADHs and the conversion was monitored (Figure $2 \mathrm{a}$ ). In the optimal case, none of these ADHs should be able to undergo such isomerization reactions. Interestingly, two of the five ADHs with $R$-selectivity in the ketoreduction showed a conversion, albeit only to a minor extent, to the lactone. It is further evident that this process is highly selective, since in both cases (P1-A04 \& P1-G03) almost exclusively the transconfigured osmundalactone $(S, R)-\mathbf{4}$ is found. When looking at the $S$-preferred ADHs instead, the exact opposite emerges. Here we find for both ADHs (NADH101 \& evo030) the cisconfigured osmundalactone $(S, S)-4$ with a diastereomeric excess of $>99 \%$. In order to gain further insights into potential ways to decouple the two dehydrogenasemediated processes, the actual cofactor specificities of all relevant ADHs were recorded (Figure 3b). Gratifyingly, the most promising ketoreductase modules all exhibited a strong NADH preference whereas the isomerase modules P1-C01 and P2-B02 showed high selectivities for NADPH as cofactor.

Before we could combine all biocatalytic sub-steps into a complete cascade consisting of reduction, oxidative ring expansion reaction and redox isomerization, we also had to further evaluate the influence of the preferred redox isomerases P1-C01 and P2-B02 on the enantioselectivity of acetylfuran 1 reduction. In the case of the reduction by $(R)$ furylethanol 2, we barely encountered any effects on the enantioselectivity by the action of two ADHs in the crossexperiments. In the worst case, an $e e$ value of $>96 \%$ (P1H10) was still obtained, whereas the best enantiomeric excess of $>99 \%$ was observed in the reaction of NADH110/P1-C01. Thus, all $R$-selective ADHs qualify for further investigation regarding the full enzyme cascade to form the osmundalactone $(S, R)$-4. If, we take a look at the $S$ selective reduction, a completely different picture is revealed. Here, the combination of two ADHs results in a drastic collapse of the enantiomeric excess. In the case of NADH101/P2-B02, only an ee value of 49\% was observed, which renders this combination useless for further studies. When evo030 is combined with P2-B02, an acceptable ee value about $74 \%$ is still achieved. Nevertheless, the prospect of a successful implementation in a one-pot reaction seems to be dizzyingly low here to obtain the cisosmundalactone $(S, S)-4$.

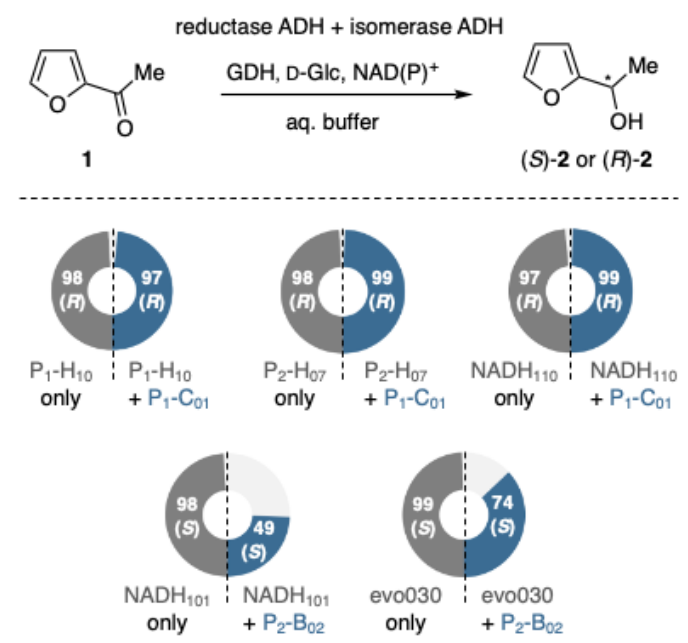

Figure 4 | Assessment of the extent of undesired interference of the designated isomerase modules (P1-C01 \& P2-B02) on the enantioselectivity of potential ketoreductase units
For the production of the trans-configured osmundalactone $(S, R)-\mathbf{4}$, we subsequently tried a one-pot process consisting of three main enzymes, the combination of two ADHs as well as CPO and their two auxiliary enzymes GDH and GOx. To our astonishment, this biocatalytic cascade seemed to work exceptionally well for the cases of P1-H10 as well as P2-H07. Already after four hours, a 50\% conversion was observed in both cases, as well as excellent enantio- and diastereoselectivity. Unpleasantly, the formation of a 5-membered ring lactone was also observed to a small extent. Performing the cascade under glucosedependent conditions with NADH110 proved tragically to be completely unsuitable, as only a conversion of just under $5 \%$ to the desired 6-ring lactone was achieved. If the first step is changed from glucose to formate, i.e. from GDH to the corresponding FDH recycling system, a conversion of almost $90 \%$ was achieved. Nevertheless, the enantiomeric and diastereomeric excess suffers with $97.5 \%$ ee and $93 \%$ de, respectively. Moreover, the formation of the undesired 5 -membered ring lactone is increased up to $10 \%$. This gave us the idea to run the enzymatic cascade in sequential addition of the individual components instead of in a onepot process. Fortunately, we thus achieved the exclusive formation of the desired trans-osmundalactone $(S, R)-\mathbf{4}$ with excellent enantio- as well as diastereoselectivity of $>99 \%$ ee and $>99 \%$ de. Moreover, the trans-configured lactone $(S, R)$ 4 could be obtained on a preparative scale with a yield of $52 \%$ by simple extraction (Figure 5 ).

a module assembly for a trans-selective osmundalactone synthesis

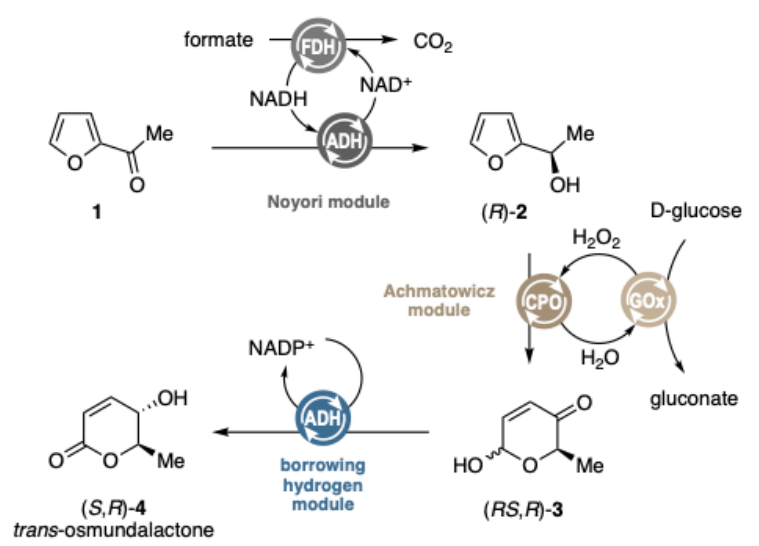

b product specificity of the modules illustrated by resulting GC traces

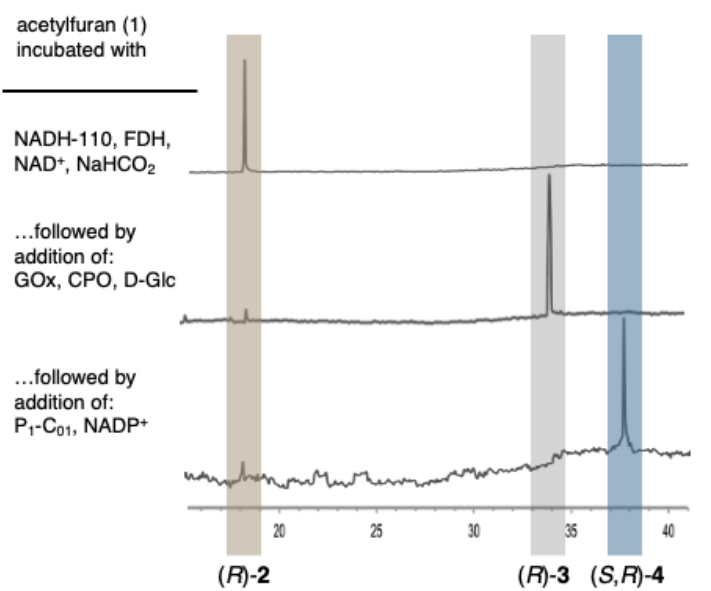

$52 \%$ isolated yield, $99 \%$ ee, $99 \%$ trans

Figure 5 | Strategy and preparative implementation for the enzymatic, chemoinspired synthesis of trans-osmundalactone 
In the case of cis-osmundalactone $(S, S)$-4 production, we opted directly for a sequential cascade reaction since it had previously been shown that the ADHs interfere with each other in opposite directions. Accordingly, we were able to obtain the desired cis-lactone $(S, S)-\mathbf{4}$ here in a glucose-dependent system by combining evo030 and P2B02 on a preparative scale with an excellent yield of $69 \%$. Even here, remarkable selectivities of $>99 \%$ ee and $>99 \%$ de were obtained.

a module assembly for a cis-selective osmundalactone synthesis

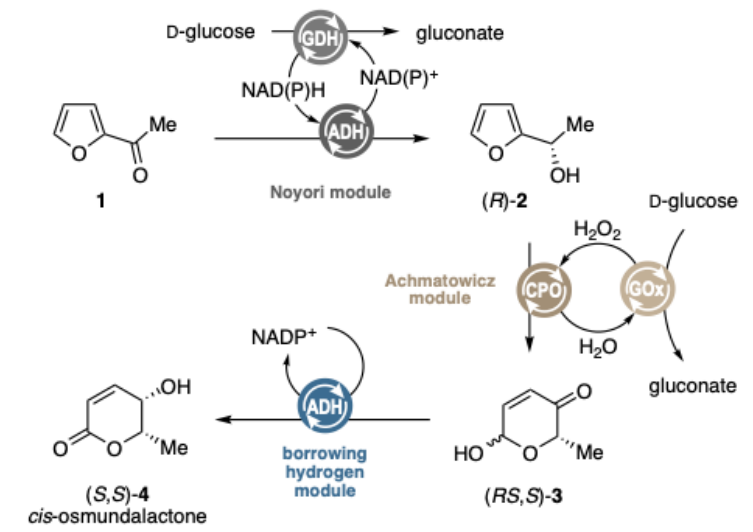

b product specificity of the modules illustrated by resulting GC traces

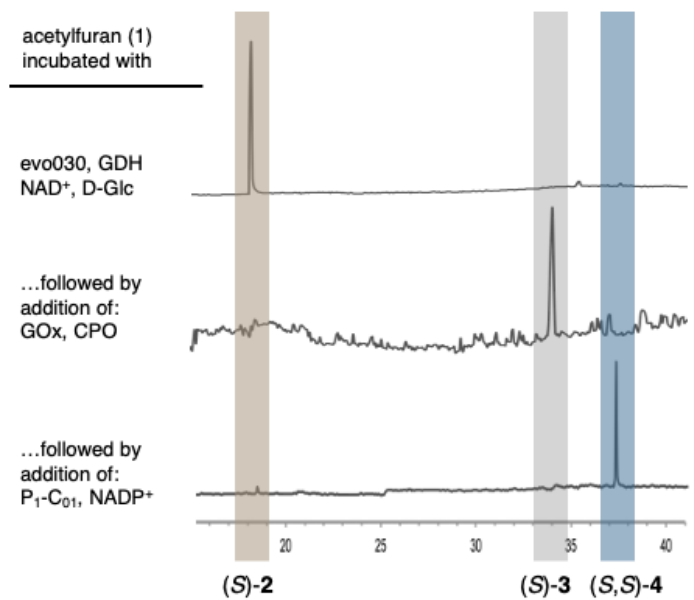

(S)-2

$(S)-3 \quad(S, S)-4$

$69 \%$ isolated yield, $99 \%$ ee, $99 \%$ cis

Figure 6 | Sequential one-pot cascade for the cis-selective biocatalytic synthesis of osmundalactone.

As the biosynthesis of the angiopterlactones has so far not been elucidated, the question arises whether a particular enzyme was responsible for the formation of the secondary metabolites, or if the dimerization occurred as a non-biosynthetic transformation inside the plant. Lawrence and coworkers could already show that traces of the tricyclic angiopterlactone B would form with catalytic amounts of an inorganic base even in aqueous media. We envisaged that under those conditions, lactone hydrolysis may lead to ring-opened carboxylates and as a result to these very low yields. To our very delight, slight variation of the aqueous dimerization protocol through addition of an acidic post-treatment led to an astounding yield of $49 \%$ of the tricyclic lactone. In order to decipher the tolerance of this method to various factors relevant for a potential implementation with the biocatalytic tools involved in the osmundalactone synthesis, a number of deviations were tested. Firstly, dimerization did in fact also take place without heating, yet at a much lower rate yielding only $12 \%$ of $\mathbf{5}$ after three days. Nevertheless, this observation does does support the assumption that at least the formation of angiopterlactone B could proceed in a spontaneous, nonbiosynthetic manner in the plant too. Under no conditions, the bicyclic angiopterlactone A could be detected, not even in traces, which still leaves questions on their synthetic relationship. In phosphate buffer, after addition of 20 mol-\% of carbonate, or in presence of excess base, also substantially reduced yields were observed. Omission of either the basic or acidic part of the treatment resulted in no detectable angiopterlactone B. Likewise, the presence of glucose fully inhibited the dimerization.

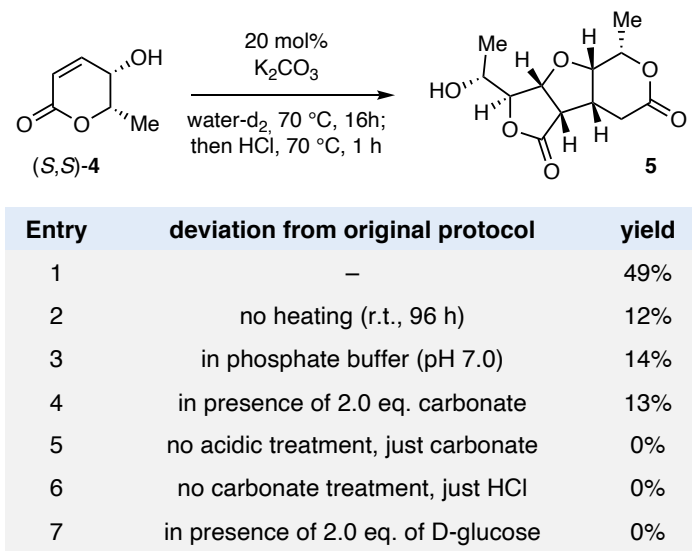

Figure 7 | Dimerization study in aqueous media

With all the different reaction modules properly assessed, and all dependencies and interferences mapped out, we were eventually able to design a sequential streamlined total synthesis protocol in which the original chemical synthesis strategy would have been entirely interpreted by a multi-enzymatic artificial metabolism, aided only by $\mathrm{pH}$ adjustments to the aqueous reaction medium. With the incompatibility of the dimerization with high concentrations of glucose, we chose to separate the sequence into two individual enzyme-driven processes. In the first step, the previously described four-enzyme biotransformation was utilized to convert acetylfuran $\mathbf{1}$ to the pyranone $(S)-3$ in greater $99 \%$ ee and greater $99 \%$ conversion. The thus obtained $(S)-3$ was subsequently treated with the isomerization biocatalyst P1-C01, followed by the herein disclosed base/acid dimerization protocol in the same aqueous reaction medium. Stunningly, the target compound angiopterlactone B (5) was thereby obtained in an overall yield of $27 \%$ (Figure 8), a more than ten-fold improvement relative to the original multi-step chemical synthesis yielding $2.3 \%$ over the four individual steps.

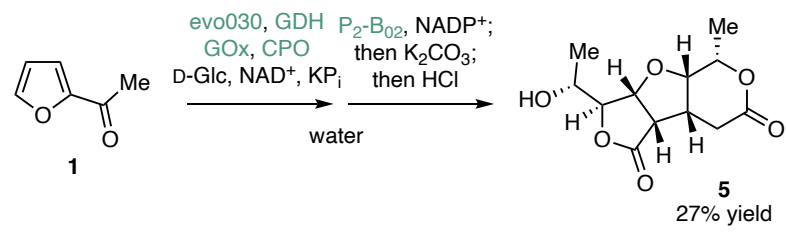

Figure 8 | A streamlined total synthesis of angiopterlactone B based on a complex biocatalytic cascade design. 
In summary, this study illustrates the enormous potential of biocatalytic cascade design in the context of organic synthesis of complex molecular architectures. By combining native and abiotic enzyme modules to an integrated metabolism-like network, great synergies are achieved and the efficacy of the process can be vastly improved even compared to the already highly elegant blueprint that provided the retrosynthetic foundation. In addition to a finely adjusted network analysis of multiple biocatalytic entities, this work sheds light on the pivotal dimerization process converting osmundalactone to the tricyclic angiopterlactone $\mathrm{B}$, both in terms of its synthetic implementation and its biosynthetic mode of action, as we disclose an effective protocol in an aqueous reaction medium with high selectivity and unmatched yields.

\section{Acknowledgements}

We gratefully acknowledge financial support of this study by the Academy of Finland (298250 (JD) \& 324854 (YCL)), the Erasmus+ programme of the European Union (RG), the Finnish Foundation for Technology Promotion and the Orion Research Foundation sr for personal grants (CJ).

\section{Keywords}

Enzyme cascade, total synthesis, lactones, asymmetric catalysis, synthetic biology

\section{References}

[1] C. A. Martinez, S. Hu, Y. Dumond, J. Tao, P. Kelleher, L. Tully, Org. Process Res. Dev. 2008, 12, 392-398.

[2] S. E. Payer, H. Pollak, B. Schmidbauer, F. Hamm, F. Juričić, K. Faber, S. M. Glueck, Org. Lett. 2018, 20, 5139-5143.
[3] F. Xu, J. Y. L. Chung, J. C. Moore, Z. Liu, N. Yoshikawa, R. S. Hoerrner, J. Lee, M. Royzen, E. Cleator, A. G. Gibson, et al., Org. Lett. 2013, 15, 1342-1345.

[4] M. A. Huffman, A. Fryszkowska, O. Alvizo, M. BorraGarske, K. R. Campos, K. A. Canada, P. N. Devine, D. Duan, J. H. Forstater, S. T. Grosser, et al., Science 2020, 368, 1255-1259.

[5] J. Liang, J. Lalonde, B. Borup, V. Mitchell, E. Mundorff, N. Trinh, D. A. Kochrekar, R. N. Cherat, G. Ganesh Pai, Org. Proc. Res. Dev. 2010, 14, 193-198.

[6] N. J. Turner, E. O'Reilly, Nat. Chem. Biol. 2013, 9, 285288.

[7] P. Dydio, H. M. Key, A. Nazarenko, J. Y.-E. Rha, V. Seyedkazemi, D. S. Clark, J. F. Hartwig, Science 2016, 354, 102-106.

[8] M. Jeschek, R. Reuter, T. Heinisch, C. Trindler, J. Klehr, S. Panke, T. R. Ward, Nature 2016, 537, 661-665.

[9] O. F. Brandenberg, C. K. Prier, K. Chen, A. M. Knight, Z. Wu, F. H. Arnold, ACS Catal. 2018, 8, 2629-2634.

[10] N. Losada-Garcia, Z. Cabrera, P. Urrutia, C. GarciaSanz, A. Andreu, J. M. Palomo, Catalysts 2020, 10, 1258-1277.

[11] J. M. Sperl, V. Sieber, ACS Catal. 2018, 8, 2385-2396.

[12] Y. M. Yu, J. S. Yang, C. Z. Peng, V. Caer, P. Z. Cong, Z. M. Zou, Y. Lu, S. Y. Yang, Y. C. Gu, J. Nat. Prod. 2009, 72, 921-924.

[13] M. I. Thomson, G. S. Nichol, A. L. Lawrence, Org. Lett. 2017, 19, 2199-2201.

[14] D. Thiel, D. Doknic, J. Deska, Nat. Commun. 2014, 5, 5278.

[15] F. Blume, Y.-C. Liu, D. Thiel, J. Deska, J. Mol. Catal. $B$ Enzym. 2016, 134, 280-284.

[16] D. Thiel, F. Blume, C. Jäger, J. Deska, Eur. J. Org. Chem. 2018, 20, 2717-2725.

[17] Y.-C. Liu, C. Merten, J. Deska, Angew. Chem. Int. Ed. 2018, 57, 12151-12156. 